\title{
Simple Model for Exploding Pusher Targets
}

\author{
Damon V. Giovanielli
}

Charles W. Cranfill 
SIMPLE MODEL FOR EXPLODING PUSHER TARGETS

by

Damon V. Giovanielli and Charles W. Cranfill

\begin{abstract}
A simple analytic model for: the behavior of thin-walled, DT-filled spherical shells irradiated with high-intensity laser light has been developed. We show that experimental results obtained with these targets can be explained weil with the model over the range of laser intensities that have been used.
\end{abstract}

\title{
I. INTRODUCTION
}

Analytic models that describe physical phenomena are preferable to numerical solutions if the analytic models can be derived while still preserving the essential physics. A limited understanding of which physical processes are dominant clouds our judgement of the validity of a given model. As data become available, the model can be used to predict the outcome of individual experiments and more important, the dependence of derived quantities on input parameters.

Models have been presented ${ }^{1}$ to explain the results of laser driven spherical exploding pusher experiments - the only spherical experiments that have been successful to date. Sufficient data now exist over a range of target and laser parameters to allow comparison to the models and lend confidence to the ability of the models to predict behavior in new parameter regimes. A model is presented below that is, perhaps, the simplest (conceptually) of those presented thus far, and appears to fit the available data at least as well as other models. A. Model Assumptions

1. A11 of the absorbed laser energy, minus the fast ion and radiative losses, goes to heating the target shell and fuel mass.

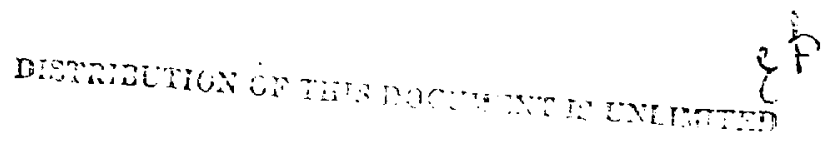


2. The energy is carried throughout the target instantaneously by energetic electrons whose ranges are large compared to target areal mass densities.

3. The fuel and pusher material are in thermal equilibrium at peak compression.

4. Half of the shell mass explodes cutward and half explodes inward pushing the suel mass in front of it.

5. 'The inner half of the shell distributes itself with uniform density from the initial sphere radius to the compressed radius.

6. The shell collapse velocity is the sound speed

$$
C_{s}=\sqrt{\frac{Z_{s}}{A_{s}} \frac{T}{M_{p}}}
$$

7. The fuel density is uniform throughout the core.

8. The fusion reaction time is twice the time it takes for the compressed fuel to expand such that the fuel ion density decreases by a factor of $\sqrt{2}$ 。

9. The fuel expansion is isothermal and occurs with a velocity equal to the sound speed in the fuel.

10. The D-T reaction rate is given by the Gamow formula.

11. Uniform heating of the target is assumed and no hydrodynamic instabjities are considered.

12. Shock heating is not included.

B. Definitions

To simplify the later discussions, a table of the symbols used in the analysis is included here:

B - laser light absorption fraction [typically 0.25 (Ref. 2)].

$\varepsilon_{i} \quad$ - fast ion and radiative loss fraction [typically 0.25 (Ref. 3)].

$E_{L}(t)$ - laser energy input up to time $t$.

$t_{b} \quad$ - time of peak compression, referenced to start of laser pulse.

$E_{s}, E_{f}$ - useful energy in shell, fuel at time $t_{b}$.

$E_{u}=E_{s}+E_{f}$.

$M_{S}, M_{f}=$ mass of shell, fuel.

$M \quad \equiv M_{s}+M_{f}$.

$e^{0} \quad \equiv E_{u} / M-$ use ${ }^{\varepsilon} u l$ specific energy.

$M_{p} \quad-$ one atomic mass unit.

$T_{s}, T_{f}$ - temperature of shell, fuel (energy units). 
$A_{s}, A_{f}$ - average atomic weights of shell, fuel.

$Z_{s}, Z_{f}$ - average ionic charge of shell, fuel.

$\rho_{s}, \rho_{f}$ - initial material density of shell, fuel.

$\mathrm{N}_{i_{s}}, N_{i_{f}}$ - number of ions in shell, fuel.

$n_{i_{s}}, n_{i_{f}}-$ ion density in shell, fuel at $t=t_{b}$.

$r_{1} \quad$ - original shell radius (center of shell).

$\Delta r_{1} \quad$ - original shell wall thickness.

$r_{0} \quad$ - collapsed fuel radius.

st - reaction duration time.

$\delta r \quad$ - fuel radius increase in time $\delta t / 2$.

C. Derivation of Expression for Yield

From assumptions 1 and 2

$$
E_{u}=\beta\left(1-\varepsilon_{i}\right) E_{L}\left(t_{b}\right)=\frac{3}{2}\left[N_{i_{s}}\left(1+Z_{s}\right) T_{s}+N_{i_{f}}\left(1+Z_{f}\right) T_{f}\right] .
$$

Solving for $\mathrm{T}_{\mathrm{S}}$

$$
T_{s}=\frac{2}{3} \frac{E_{u}}{M} \frac{A_{s}}{1+Z} M_{p} \frac{1+\frac{M_{f}}{M_{s}}}{1+\frac{M_{f}}{M_{s}} \frac{A_{s}\left(1+Z_{f}\right)}{A_{f}\left(1+Z_{s}\right)} \frac{T_{f}}{T_{s}}} \text {. }
$$

From assumption $3, T_{S}=T_{f}$ and

$$
T_{f}=\frac{2}{3} \varepsilon \frac{A_{s}}{1+Z_{s}} M_{p} \frac{1+\frac{M_{f}}{M_{s}}}{1+\frac{M_{f}}{M_{s}} \frac{A_{s}}{A_{f}} \frac{\left(1+Z_{f}\right)}{\left(1+Z_{s}\right)}} .
$$

Using assumption 3 and the fact that at peak compression the fuel and pusher pressures are equal (i.e., momentum is conserved),

$$
\left(1+z_{f}\right) n_{i_{f}}=\left(1+z_{s}\right) n_{i_{s}}\left(t=t_{b}\right) .
$$

Then from assumptions 4 and 5 :

$$
n_{i_{s}}=\frac{1}{2} \frac{N_{i_{s}}}{4 \pi r_{1}^{2}} \frac{3 r_{1}^{2} \Delta r_{1}}{r_{1}^{3}-r_{0}^{3}}=\frac{3}{8 \pi} \frac{M_{s}}{A_{s} M_{p}} \frac{1}{r_{1}^{3}-r_{0}^{3}} .
$$


From as sumption 7 :

$$
n_{i_{f}}=\frac{3}{4 \pi} \frac{M_{f}}{A_{f} M_{p}} \frac{1}{r_{o}^{3}} .
$$

Combining Eqs. (2), (3), and (4) we obtain the collapsed fuel radius $r_{0}$ in terms of the target parameters,

$$
r_{0}=\left(1+\frac{1}{2} \frac{1+Z_{s}}{1+Z_{f}} \frac{A_{f}}{A_{s}} \frac{M_{s}}{M_{f}}\right)^{-1 / 3} r_{1} .
$$

We see that for a given material shell density $\rho_{s}$ and a given initial gaseous fuel fill pressure $\left(\rho_{f}=\rho_{o} P\right)$

$$
r_{0}=\left(1+\frac{3}{2} \frac{1+Z_{s}}{1+Z_{f}} \frac{A_{f}}{A_{s}} \frac{\rho_{s}}{\rho_{f}} \frac{\Delta r_{1}}{r_{1}}\right)^{-1 / 3} r_{1} .
$$

For the special case given by $\rho_{s} / \rho_{f}=10^{4} / P(A T M), A_{f}=2.5, A_{s}=20, z_{s}=10$, $\mathrm{Z}_{\mathbf{f}}=1$,

$$
r_{0} \approx\left(1+\frac{10^{4}}{\mathrm{P}(\mathrm{ATM})} \frac{\Delta \mathrm{r}_{1}}{\mathrm{r}_{1}}\right)^{-1 / 3} \mathrm{r}_{1}
$$

The collapse time $t_{b}$ is obtained from Eqs. (1) and (5) and assumption 6

$$
t_{b}=\frac{r_{1}-r_{0}}{C_{s}} \text {. }
$$

The time during which fusion reactions take place is, using assumption 8 and 9 ,

$$
\delta t=\frac{2 \delta r}{\sqrt{\frac{Z_{f}}{A_{f}} \frac{T_{f}}{M_{p}}}},
$$

where $\delta \mathbf{r}$ is obtained, under assumption 8 , from

$$
\left(\frac{r_{0}+\delta r}{r_{0}}\right)^{3}=\sqrt{2}
$$


or

$$
\delta \mathrm{r}=\mathrm{r}_{\mathrm{o}}\left(2^{1 / 6}-1\right)=0.1225 \mathrm{r}_{\mathrm{o}}
$$

The volume within which reactions are assumed to take place is taken as the average of the minimum compressed volume and the volume at which point $n_{i_{f}}$ drops by $\sqrt{2}$,

$$
V=\frac{1}{2} \frac{4}{3} \pi r_{0}^{3}(1+\sqrt{2})=5.06 r_{0}^{3}
$$

The fusion yield from the target is obtained from the formula.

$$
Y=n_{i_{f}}{ }^{2} V \delta t \overline{\sigma v}
$$

The average emission volume, $V$, is given by Eq. (12); the burn duration is given by Eq. (9), using Eqs. (11) and (6). The value of $\mathrm{n}_{\mathrm{i}_{\mathrm{f}}}{ }^{2}$ is taken as the peak value (at minimum fuel radius), and $\overline{\sigma v}$ is evaluated at the temperature of the fuel at peak compression.

Combining Eqs. (12), (9), (11), (6), (4), and the Gamow formula for the D-T

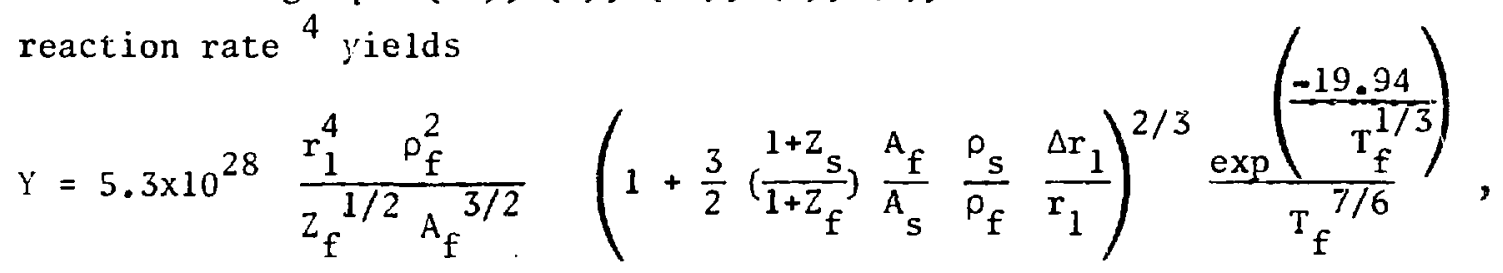

where $T_{f}$ is in $\mathrm{keV}$, length in $\mathrm{cm}$, and initial densities in $\mathrm{g} / \mathrm{cm}^{3}$. In the case of moderate aspect ratios (less than 300) and an initial fuel density to shell density ratio less than $10^{-3}$, then the second term in the parentheses in Eq. (14) dominates and

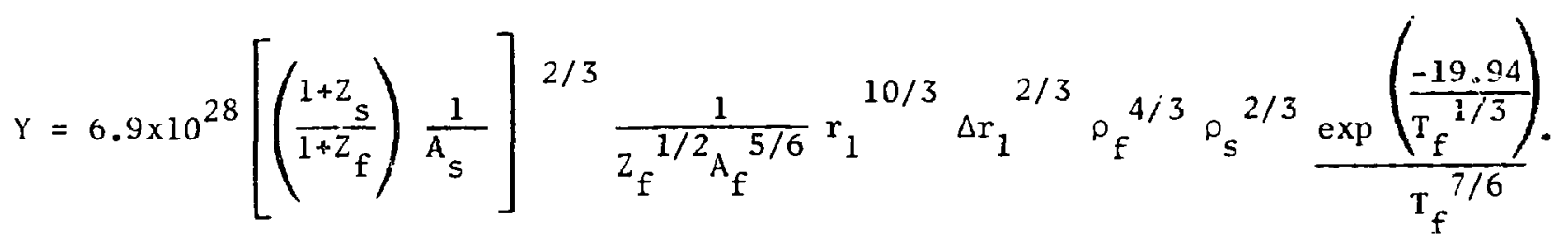

For a DT fuel, $z_{f}=1, A_{f}=2.5$. The ratio $\left(1+z_{s}\right) / A_{s}$ for any pusher material is approximately 0.5 which leaves 


$$
Y \cong 1.3 \times 10^{28} \mathrm{r}_{1}^{10 / 3} \Delta \mathrm{r}_{1}{ }^{2 / 3} \rho_{\mathrm{f}}^{4 / 3} \rho_{\mathrm{S}}^{2 / 3} \exp \left(\frac{-19.94}{\frac{\mathrm{T}_{\mathrm{f}}^{1 / 3}}{\mathrm{~T}_{\mathrm{f}}{ }^{7 / 6}}}\right),
$$

if

$$
\frac{\rho_{\mathrm{s}}}{\rho_{\mathrm{f}}}>\frac{\mathrm{r}_{1}}{\Delta \mathrm{r}_{1}}
$$

Thus, we see that each target parameter (wall thickness, radius, and DT fill pressure) has an important effect on the fusion yield, and the outputs from various targets cannot be compared without taking account of the target parameters, as well as the laser parameters, such as total energy, pulse risetime, and peak power. From Eq. (16) we see that a natural yield parameter to consider is the normalized yield $Y^{*}$, where

$$
Y^{*}=Y \times\left[\left(\frac{r^{*}}{r_{1}}\right)^{10 / 3}\left(\frac{\Delta r^{*}}{\Delta r_{1}}\right)^{2 / 3}\left(\frac{\rho_{f}^{*}}{\rho_{f}}\right)^{4 / 3}\left(\underset{\rho_{s}}{\rho_{s}^{*}}\right)^{2 / 3}\right],
$$

and $r^{*}, \Delta r^{*}, \rho_{f}^{*}, \rho_{s}^{*}$ are convenient normalization values for initial radius and wall thickness and initial fuel and shell mass densities. A convenient separation of parameters then occurs if $\mathrm{T}_{f}$ depends only on the laser parameters but not (or only weakly) on the initial target parameters.

If condition (17) is satisfied, then

$$
\frac{M_{f}}{M_{s}}=\frac{1}{3} \frac{\rho_{f}}{\rho_{s}} \frac{r_{1}}{\Delta r_{1}} \ll 1,
$$

and Eq. (1), for the fuel temperature during burn becomes

$$
\mathrm{T}_{\mathrm{f}} \cong \frac{2}{3} \frac{\mathrm{A}_{\mathrm{s}} \mathrm{M}}{1+Z_{s}} \varepsilon
$$

or

$$
T_{f}(k e V) \approx \frac{7 A_{s}}{1+Z_{s}} \quad E(J / n g)
$$


Unfortunately, $\varepsilon^{\circ}$, the useful specific energy, or the useful absorbed ene'gy per unit target mass, depends both on the laser parameters and the target parameters. Since $\varepsilon^{e}$ is the absorbed laser energy, minus fast ion and radiative losses, per unit target mass, integrated up to peak compression time $t_{b}$, the target dimenisions and initial densities must enter;

$$
\varepsilon=\beta\left(1-\varepsilon_{i}\right) \frac{E_{L}\left(t_{b}\right)}{N}
$$

For a triangular temporal profile to the laser pulse, which rises linearly from zero to peak power in time $\tau_{1}$ and falls linearly back to zero in time $\tau_{2}$, the base-to-base temporal width is $\tau_{1}+\tau_{2}$ and the FWHM is $1 / 2\left(\tau_{1}+\tau_{2}\right)$. If the peak power is $P_{0}$, then the total energy in the laser pulse striking the target is $E_{0}=0.5 \times P_{o} \times\left(\tau_{1}+\tau_{2}\right)$ and

$$
\begin{aligned}
& \frac{E_{0} t^{2}}{\tau_{1}\left(\tau_{1}+\tau_{2}\right)}, \quad 0<t<\tau_{1} \\
& E_{L}(t)= \begin{cases}\frac{2 E_{0}}{\tau_{2}\left(\tau_{1}+\tau_{2}\right)}\left[\left(\tau_{1}+\tau_{2}\right)\left(t-\frac{\tau_{1}}{2}\right)-\frac{\tau^{2}}{2}\right], \tau_{1}<t<\tau_{1}+\tau_{2} \\
E_{0}, t>\tau_{1}+\tau_{2} .\end{cases}
\end{aligned}
$$

$E$ must be found by iteration; the useful energy needed to cause the inner half of the shejl to move into the radius $r_{0}$ at time $t_{b}$ must be equal to the useful laser energy available up to that time. For cases such that conditions (17) and (19) are satisfied, we can use Eqs. (6), (8), and (20) to get the approximate expression

$$
t_{b} \approx 1.22\left[1-\left(\frac{\rho_{f}}{\rho_{s}} \frac{r_{1}}{\Delta r_{1}}\right)\right] r_{1} / \sqrt{\frac{z_{s}}{1+z_{s}} \varepsilon} .
$$

Since $\mathcal{E}$ is derivatle from observed quantities for each target, then various experiments can be compared on a curve of normalized yield versus useful specific energy. Alternatively, normalized yield may be graphed against fuel temperature (easily derivable from $E^{\circ}$ ). 
Figure 1 shows normalized neutron yield versus useful specific energy, with the results of a number of experiments included. The experiments were performed with a $\mathrm{CO}_{2}$ laser whose parameters were

$$
\begin{aligned}
150 & <\mathrm{E}_{\mathrm{o}}<600 \mathrm{~J} \\
160<\tau_{1} & <500 \mathrm{ps} \\
0.11<\mathrm{P}_{\mathrm{o}} & <0.44 \mathrm{TW} .
\end{aligned}
$$

The ranges of target parameters covered in these experiments were

$$
\begin{gathered}
45<\mathrm{r}_{1}<150 \mu \mathrm{m} \\
0.6<\Delta \mathrm{r}_{1}<1.4 \mu \mathrm{m} \\
0.18<\rho_{\mathrm{f}}<7.2 \mathrm{mg} / \mathrm{cm}^{3} \\
\rho_{\mathrm{s}}=2.4 \mathrm{~g} / \mathrm{cm}^{3} .
\end{gathered}
$$

The agreement between the simple model and experiment is generally within experimental accuracy. Experiments quoted in Ref. 1 (Storm et al.) have also been calculated with this model and the measured yield was calculated within the quoted experimental uncertainties. Somic of these are indicated in Fig. 1.

The possibilities of predicting the scaling of yield with various target and and laser parameters, and of optimizing target designs (with respect to yield) for given laser parameters, are the real advantages of an analytic model. At least, we would hope to be able to outline the region of parameter space to be investigated by a more complete and complicated calculation.

Figures 2, 3, and 4 show the predicted variation of yield with initial carget radius, wall thickness, and DT fill pressure, respectively, other parameters being held fixed. For these cases a single temporal profile was assumed for the laser pulse. In Fig. 5 the variation of optimum DT fill pressure with laser pulse risetime is shown. In this case a shell radius of $200 \mu \mathrm{m}$ was assumed with a wall thickness of $1 \mathrm{\mu m}$.

Figure 6 shows the results of reoptimizing the target parameters at each peak laser power for two laser pulse temporal shapes. The wall thickness was constrained to be greater than $1000 \mathrm{~A}$. It is clear that breakeven (fusion energy equal to laser pulse energy) is not feasible with exploding pusher targets and typically shaped laser pulses. In fact, other laser pulse shapes also do not give breakeven results, even for rather unrealistic shapes.

Figure 7 shows the variation of yield with peak laser power for several physically realisable target geometries and pulse shapes。 


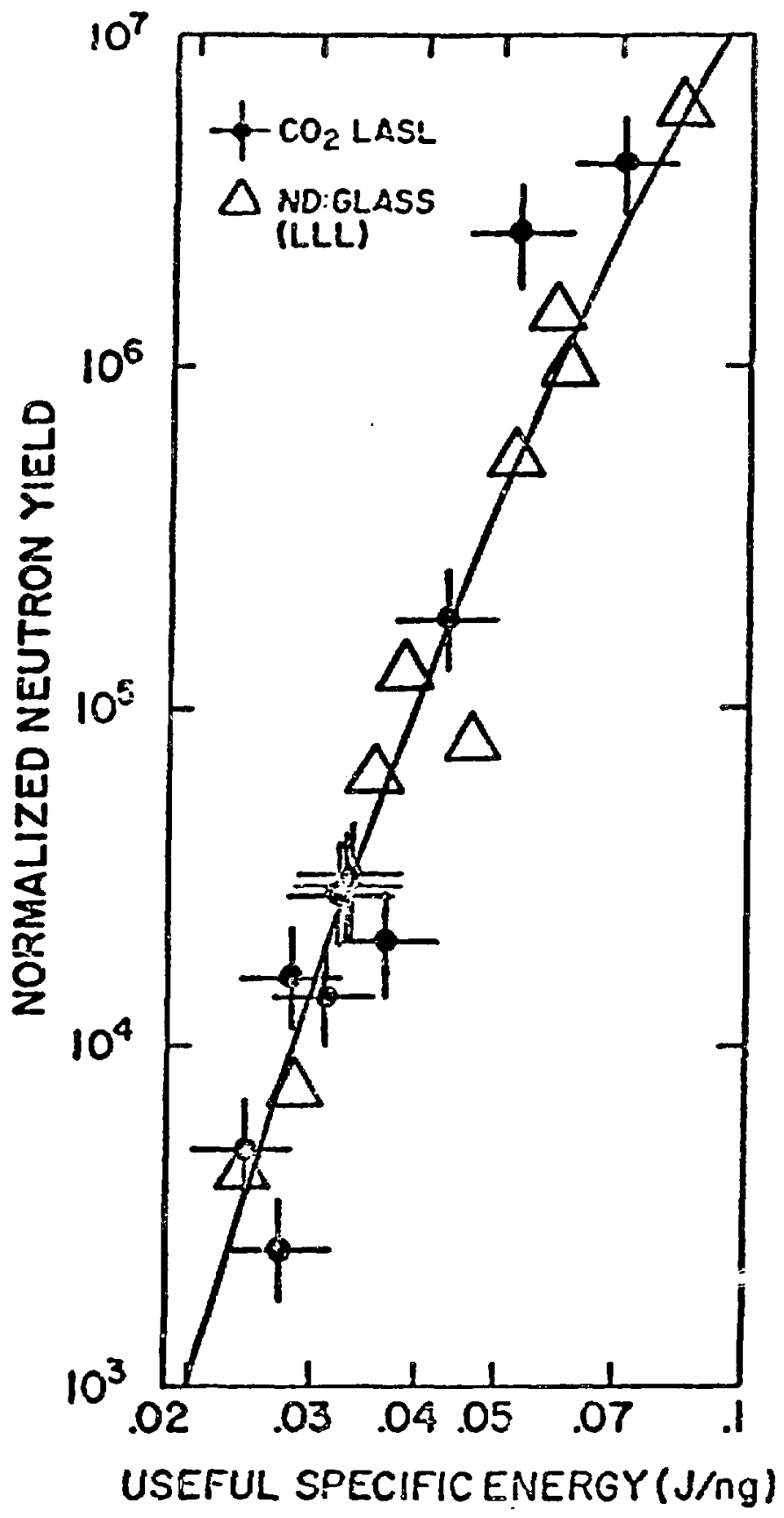

Fig. 1

Normalized neutron yield versus useful specific erergy for exploding pusher targets. Normalized yield is definged by Eq. (18) with $\mathrm{r}^{\star}=100 \mu \mathrm{m}, \Delta \mathrm{r}^{*}=1.0 \mathrm{um}$, $\rho_{\mathrm{f}^{*}}=0.2 \mathrm{mg} / \mathrm{cm}^{3}, \rho_{\mathrm{s}}^{*}=2.4 \mathrm{~g} / \mathrm{cm}^{3}$. Results of experiments using a $\mathrm{CO}_{2} 1 \mathrm{aser}(\bullet \mathrm{j}$ and a Nd:glass laser $(\Delta)$ are shown. The Nd:glass laser data are taken from $R \in \hat{f}$. 1 (Storm et al.). 


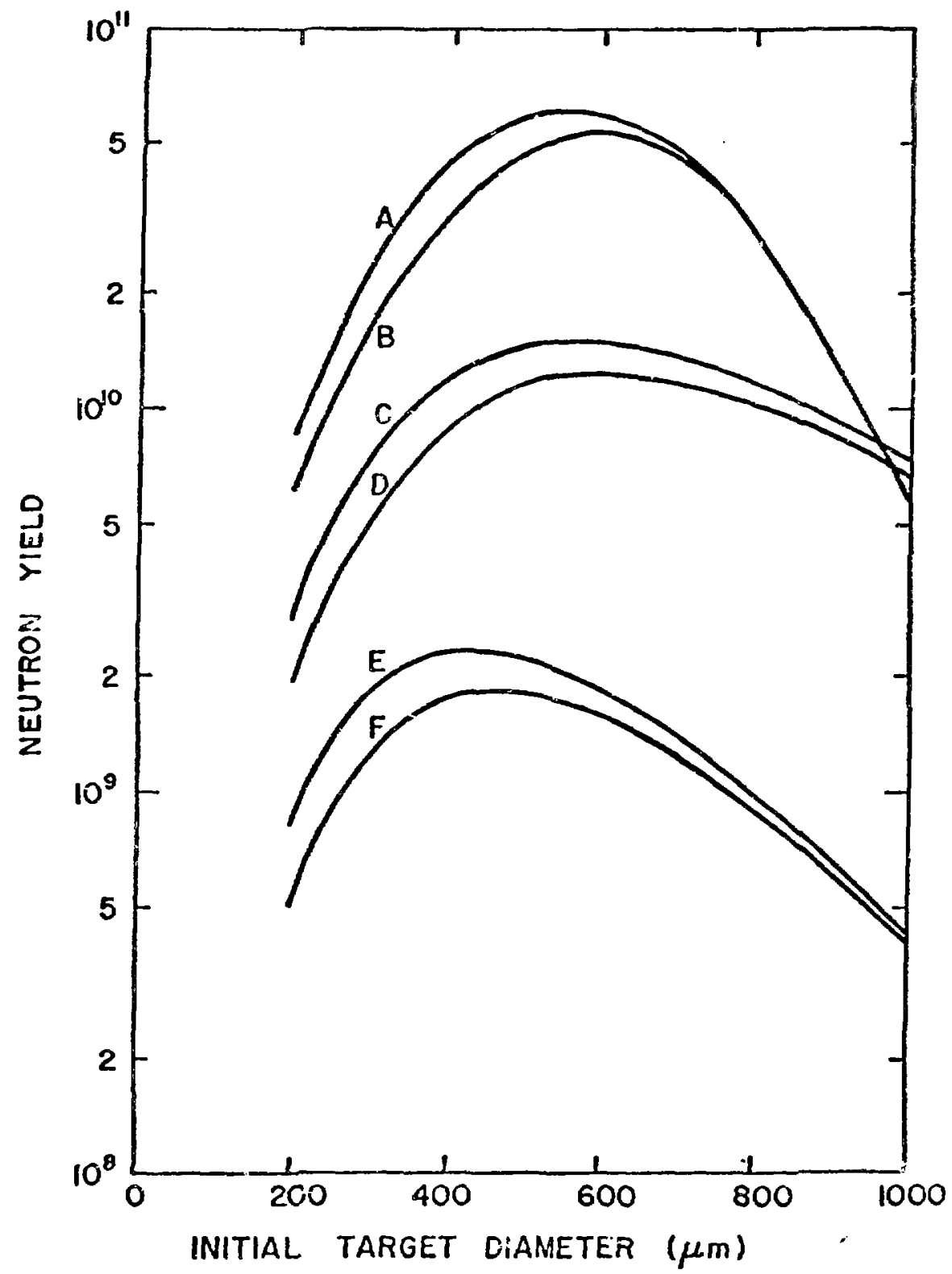

Fig. 2

Yield versus glass microballoon initial diameter for different laser parameters. Initial wall thickness was $1.0 \mu \mathrm{m}$ and DT gas fill pressure was 10 ATM. The fraction of incident laser light absorbed was taken as 0.2. Triangular temporal laser pulse shapes were used with risetime $\tau_{1}$, fall time $\tau_{2}$, peak power $P_{0}$, and totaI energy $\mathrm{E}_{\mathrm{O}}$. $\mathrm{A}: \tau_{1}=0.2 \mathrm{~ns}, \tau_{2}=0.3 \mathrm{~ns}, \mathrm{P}_{\mathrm{C}}=20 \mathrm{TW}, \mathrm{E}_{\mathrm{O}}=5 \mathrm{~kJ} ; \mathrm{B}: \tau_{1}=\tau_{2}=0.25$ $\mathrm{ns}, \mathrm{P}_{\mathrm{O}}=20 \mathrm{TW}, \mathrm{E}_{\mathrm{O}}=5 \mathrm{~kJ} ; \mathrm{C}: \tau_{1}=0.2 \mathrm{~ns}, \tau_{2}=1.8 \mathrm{~ns}, \mathrm{P}_{\mathrm{O}}=10 \mathrm{TW}, \mathrm{E}_{0}=10 \mathrm{~kJ}$; D: $\tau_{1}=0.25 \mathrm{~ns}, \tau_{2}=1.75 \mathrm{~ns}, \mathrm{P}_{\mathrm{O}}=10 \mathrm{TW}, \mathrm{E}_{\mathrm{O}}=10 \mathrm{~kJ} ; \mathrm{E}: \tau_{1}=0.2 \mathrm{~ns}, \tau_{2}=1.8$ $\mathrm{ns}, \mathrm{P}_{\mathrm{O}}=5 \mathrm{TW}, \mathrm{E}_{\mathrm{O}}=5 \mathrm{~kJ} ; \mathrm{F}: \tau_{1}=0.25 \mathrm{~ns}, \tau_{2}=1.75 \mathrm{~ns}, \mathrm{P}_{\mathrm{O}}=5 \mathrm{TW}, \mathrm{E}_{\mathrm{O}}=5 \mathrm{~kJ}$; $\mathrm{F}: \tau_{1}=0.25 \mathrm{~ns}, \tau_{2}=1.75 \mathrm{~ns}, \mathrm{P}_{\mathrm{O}}=5 \mathrm{TW}, E_{\mathrm{O}}=5 \mathrm{~kJ}$. The initial target radius for optimum yield in these cases is between $200 \mu \mathrm{m}$ and $325 \mu \mathrm{m}$ with a rather weak dependence (less than factor of 2) variation in yield over this range of radii. 


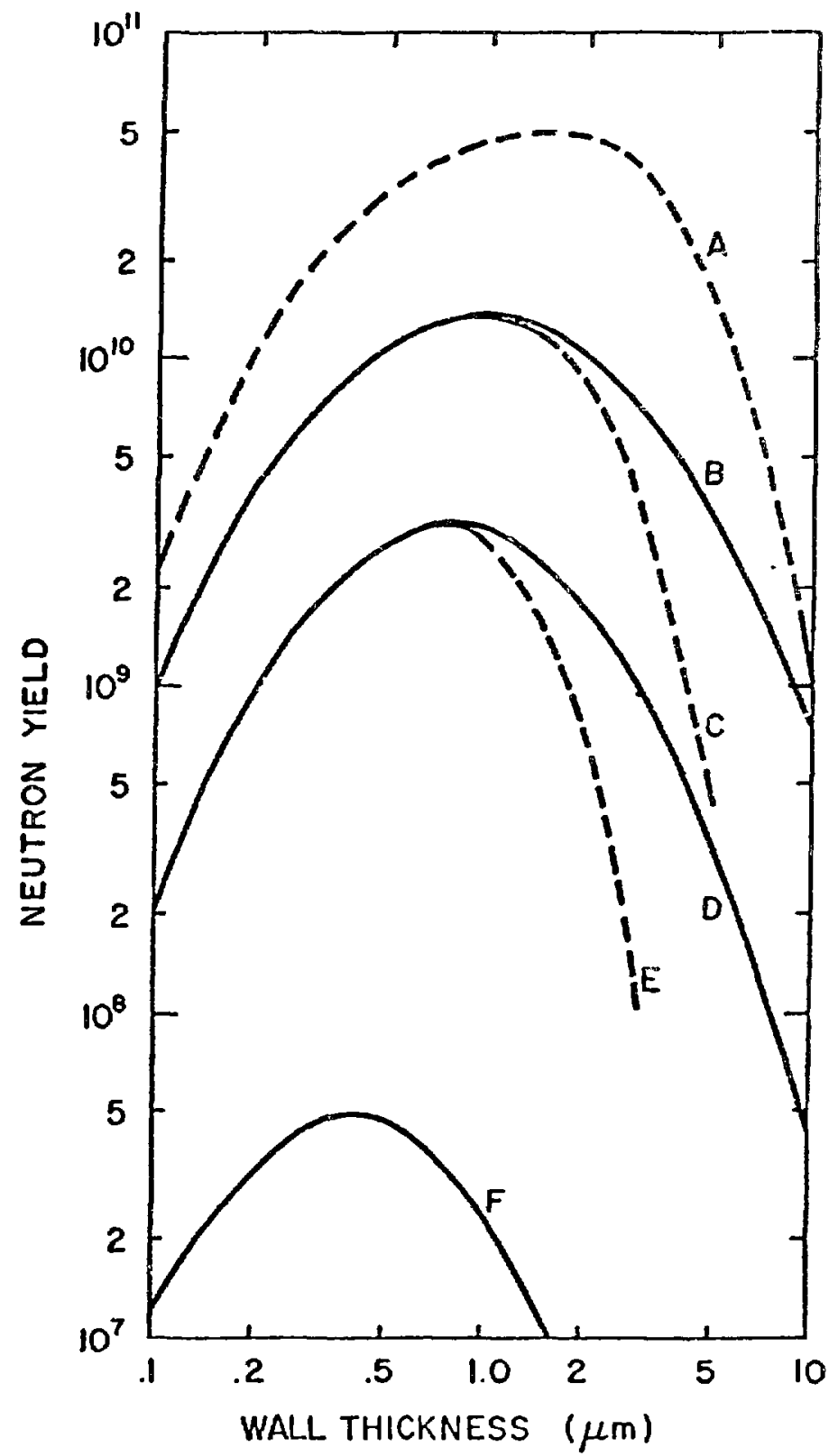

Fig. 3

Neutron yield as a function of glass microballoon wall thickness for several laser triangular temporal pulse shapes, peak laser powers, and initial target radii. The fraction of incident laser energy absorbed was 0.25 . A: $\tau_{1}=0.25 \mathrm{~ns}, \tau_{2}=0.25$ $\mathrm{ns}, \mathrm{E}_{\mathrm{O}}=5 \mathrm{~kJ}, \mathrm{P}_{\mathrm{O}}=20 \mathrm{TW}, \mathrm{r}_{1}=200 \mu \mathrm{m}$, initial DT fill pressure of $13 \mathrm{ATM}$; $\mathrm{B}$ : $\tau_{1}=0.25 \mathrm{~ns}, \tau_{2}=1.75 \mathrm{~ns}, E_{0}=10 \mathrm{~kJ}, P_{0}=10 \mathrm{TW}, r_{1}=200 \mathrm{\mu m}$, fill pressure $=$ $10 \mathrm{ATN} ; \mathrm{C}: \tau_{1}=0.25 \mathrm{~ns}, \tau_{2}=0.25 \mathrm{~ns}, E_{0}=2.5 \mathrm{~kJ}, \mathrm{P}_{0}=10 \mathrm{Th}, \mathrm{r}_{1}=200 \mu \mathrm{m}, \mathrm{fiil}$ pressure $=1 \mathrm{G} A T M ; D: \tau_{1}=0.25 \mathrm{~ns}, \tau_{2}=1.75 \mathrm{~ns}, E_{0}=5 \mathrm{~kJ}, \mathrm{P}_{\mathrm{O}}=5 \mathrm{TW}, \mathrm{r}_{1}=200$ $\mu \mathrm{m}$, fill pressure $=10 \mathrm{ATM} ; \mathrm{E}: \tau_{1}=0.25 \mathrm{~ns}, \tau_{2}=0.25 \mathrm{~ns}, E_{0}=1.25 \mathrm{~kJ}, \mathrm{P}_{0}=5$ $\mathrm{TW}, \mathrm{r}_{1}=200 \mathrm{\mu m}$, fill pressure $=10 \mathrm{ATM} ; \mathrm{F}: \tau_{1}=0.25 \mathrm{~ns}, \tau_{2}=1.75 \mathrm{~ns}, \mathrm{E}_{\mathrm{O}}=1.0$ $\mathrm{kJ}, \mathrm{P}_{0}=1 \mathrm{TW}, \mathrm{r}_{1}=150 \mu \mathrm{m}$, fill pressure $=7 \mathrm{ATM}$. 


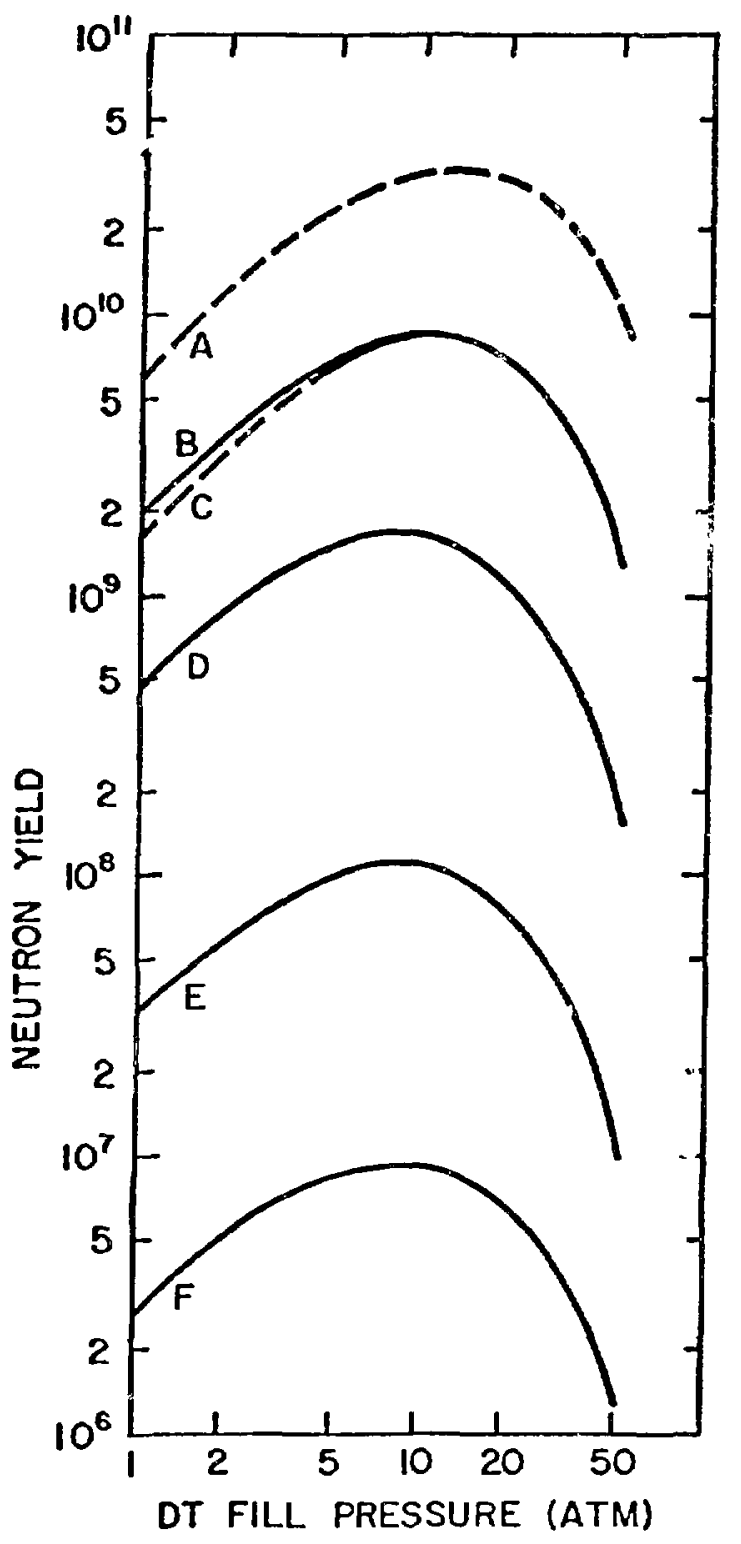

Fig. 4

Neutron yield as a function of initial DT fili pressure in a glass microballoon. The wall thickness was chosen as $\Delta \mathbf{r}_{1}=1.0 \mathrm{\mu m}$, and the zero to peak power risetime $\left(\tau_{1}\right)$ of the laser temporal pulse was taken as $0.25 \mathrm{~ns}$ in all cases. The solid curves are for a peak power to zero fall time ( $\left.\tau_{2}\right)$ of 1.75 ns, while for the dashed curves $\tau_{2}=0.25 \mathrm{ris} . A: r_{1}=200 \mu \mathrm{m}, E_{0}=5 \mathrm{~kJ}, P_{0}=20 \mathrm{TW} ; \mathrm{B}: r_{1}=$ $200 \mathrm{\mu m}, E_{O}=10 \mathrm{~kJ}, P_{O}=10 \mathrm{TW} ; C: r_{1}=200 \mu \mathrm{m}, E_{O}=2.5 \mathrm{~kJ}, P_{O}=10 \mathrm{TW} ; \mathrm{D}: r_{1}=$ $200 \mu \mathrm{m}, E_{O}=5 \mathrm{~kJ}, P_{O}=5 \mathrm{TW} ; E: r_{1}=150 \mu \mathrm{m}, E_{O}=2 \mathrm{~kJ}, P_{O}=2 \mathrm{TW} ; \mathrm{F}: r_{1}=100$ $\mu \mathrm{m}, E_{\mathrm{O}}=1 \mathrm{~kJ}, \mathrm{P}_{\mathrm{O}}=1 \mathrm{TW}$. 


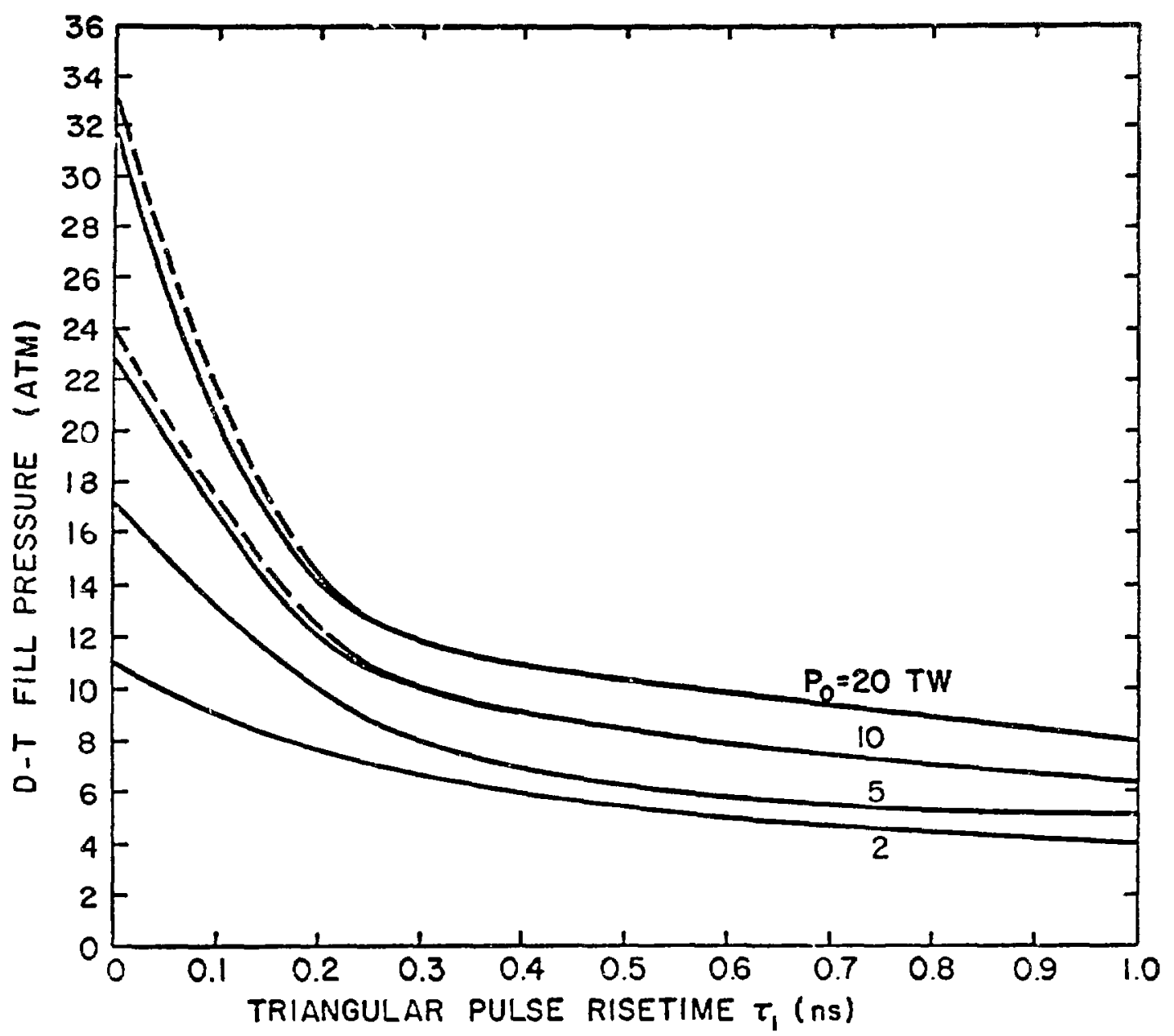

Fig. 5

Initial DT gas fill pressure (50\% each by number with no impurities) for maximum yield versus triangular laser pulse risetime. The initial glass microballoon radius was $200 \mu \mathrm{m}$ and initial wall thickness $1.0 \mu \mathrm{m}$. Solid curves are for a $1.0 \mathrm{~ns}$ FWHM laser pulse; dashed curves are for a 0.25 FWHM pulse. Peak laser powers are indicated for the four powers considered. The absorption fraction for the laser light was assumed to be 0.25 . 


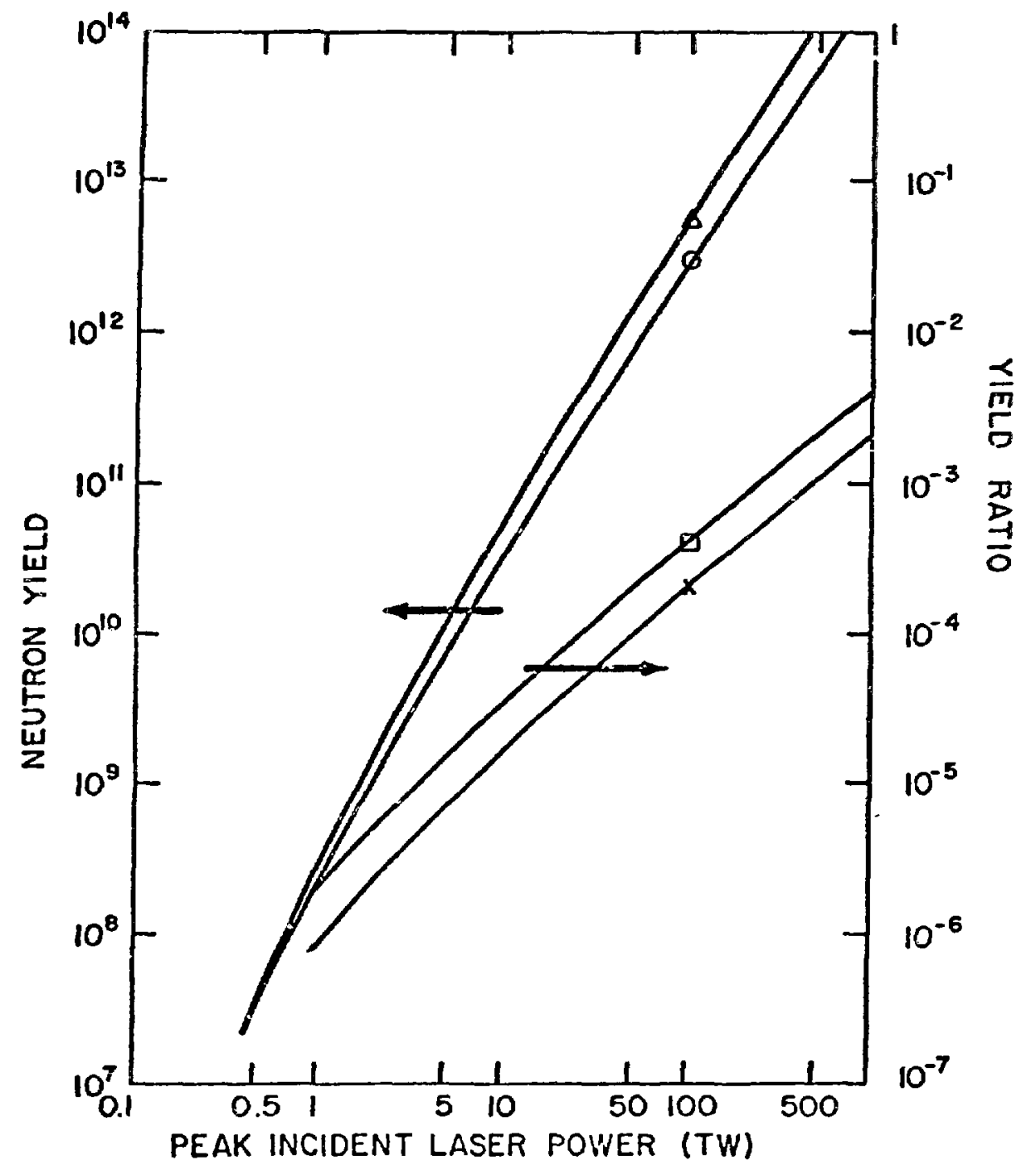

Fig. 6

Neutron yield and yield ratio (fusion energy output divided by laser energy incident) for optimized exploding pusher targets. Triangular temporal laser pulse shapes were used with a base to peak power time of $0.25 \mathrm{~ns}$. Two fall times were used leading to full widths at half maxima of 1.0 and $0.25 \mathrm{~ns} . \Delta$ : neutron yields versus peak laser power for 1.0 ns FWHM laser pulses. 0: neutron yields versus peak laser power for 0.25-ns FWHM pulses. $\square$ : yield ratio for 0.25-ns FWHM pulses. $\mathrm{X}$ : yield ratio for $1.0 \mathrm{~ns}$ FWHM. The optimized exploding pusher fusion yicld is seen to scale approximately as the square of the peak laser power. For these optimization calculations the DT gas filled glass microballoon was constrained to have a wall thickness greater than $0.1 \mu \mathrm{m} ; 25 \%$ of the laser energy was assumed to be absorbed by the target. 


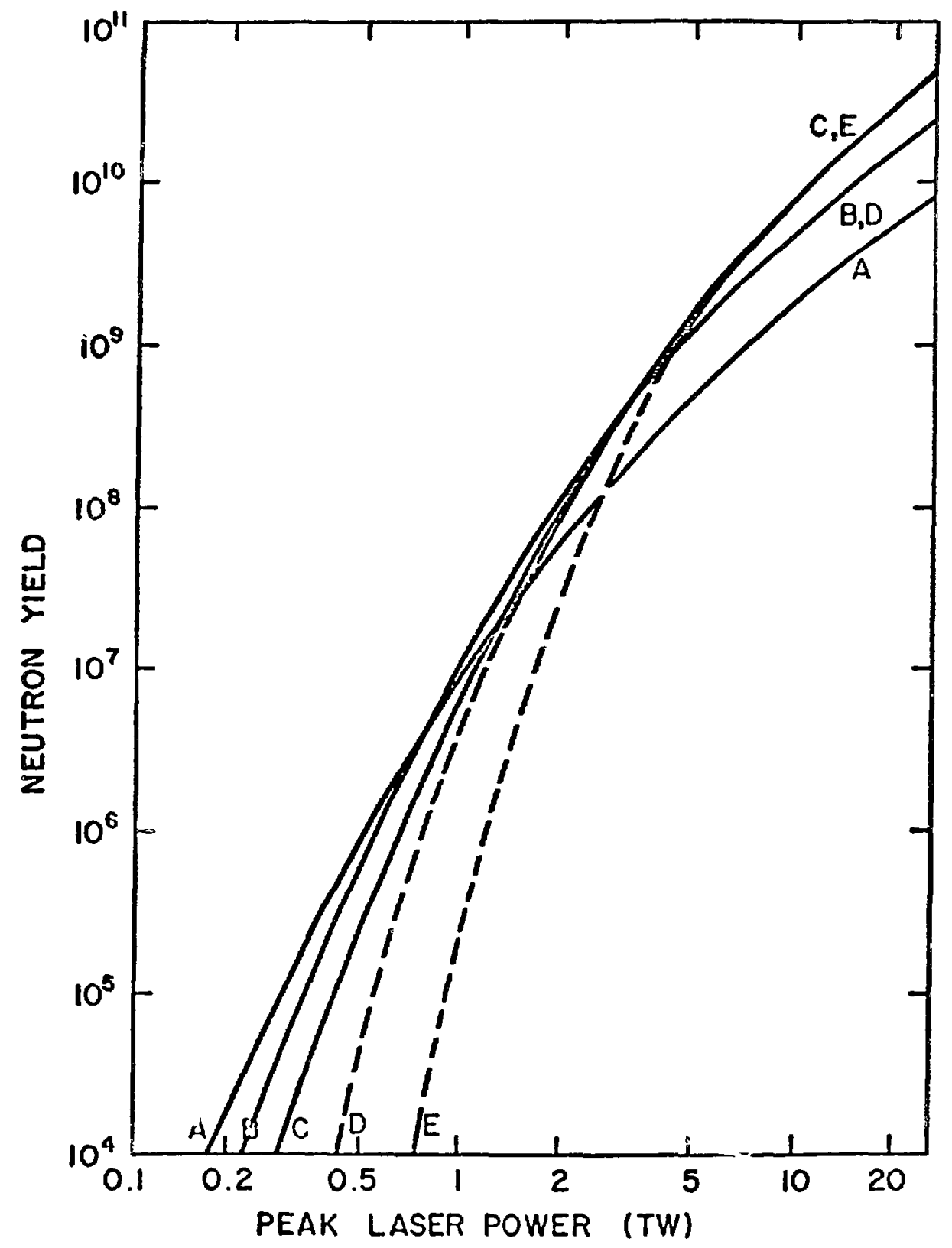

Fig. 7

Neutron yield as a function of peak incident laser power. Triangular temporal laser pulses were assumed with a base to peak power time ( $\left.\tau_{1}\right)$ of $0.25 \mathrm{~ns}$. Solid curves assume a peak power to zero fall time $\left(\tau_{2}\right)$ of $1.75 \mathrm{~ns}$; dashed curves assume a fall time of $0.25 \mathrm{~ns}$. The laser light absorption fraction was taken to be 0.2. Initial DT fill pressure was taken as 10 ATM for all cases and the initial wall thickness of the glass microballoon was taken to be $\Delta \mathrm{r}_{1}=1.0 \mu \mathrm{m}$. $\mathrm{A}: \mathrm{r}_{1}=150 \mu \mathrm{m} ; \mathrm{B}, \mathrm{D}: \mathrm{r}_{1}=150 \mu \mathrm{m} ; \mathrm{C}, \mathrm{E}: \mathrm{r}_{1}=200 \mu \mathrm{m}$. 


\section{REFERENCES}

1. R. K. Osborn and F. J. Mayer, Amer. Jour. Phys. 44, 149 (1976); E. K. Storm, J. T. Larsen, J. H. Nuckolls, and H. G. Ahlstrom, Bull。Am. Phys. Soc。22, 1086 (1977)。

2. V. Cottles, Bull。Am. Phys。Soc, 22, 1090 (1977).

3. F. Young and G. H. McCall, Bull. Am。Phys.Soc. 22, 1129 (1977).

4. S. Glasstone and R. H. Lovberg, Controlled Thermonuclear Reactions (D. Van Nostrand Company, Inc., 1960) p. 20. 